\title{
AN EXTENSION OF THE RIESZ-HERGLOTZ FORMULA
}

\author{
W. K. HAYMAN and B. KORENBLUM
}

1. Introduction and statement of results. Let

$$
g(z)=\sum_{1}^{\infty} a_{n} z^{n}=u+i v
$$

be regular in $|z|<1$. If

$$
\int_{0}^{2 \pi}\left|u\left(r e^{i \vartheta}\right)\right| d \vartheta=O(1)
$$

as $r \rightarrow 1$, or equivalently if $u$ is the difference of 2 positive harmonic functions it is classical*, that $g(z)$ possesses a representation

$$
g(z)=\frac{1}{2 \pi} \int_{0}^{2 \pi} \frac{e^{i \vartheta}+z}{e^{i \vartheta}-z} d \mu(\vartheta),
$$

where $\mu(\vartheta)$ is a function of bounded variation in $[0,2 \pi]$. In this paper we consider conditions under which $g(z)$ possesses a representation (1.2) where $\mu(\vartheta)$ is a function which is bounded, but not necessarily of bounded variation. In this case the integral (1.2) can be defined by integration by parts. Since $g(0)=0$, we deduce that $\mu(0)=\mu(2 \pi)$, so that

$$
g(z)=\frac{i}{\pi} \int_{0}^{2 \pi} \frac{z e^{i \vartheta} \mu(\vartheta) d \vartheta}{\left(e^{i \vartheta}-z\right)^{2}} .
$$

If (1.2) exists as a Riemann - Stieltjes integral, and in particular if $\mu(\vartheta)$ has bounded variation, (1.2) and (1.3) are certainly equivalent.

Next we note that if

* See e.g. [2, Theorem 1.1, p. 2]. The name Riesz-Herglotz formula has been suggested in conversation by Professor Heins. 


$$
G(z)=\frac{1}{i} \int_{0}^{z} \frac{g(\xi) d \xi}{\xi}=U+i V
$$

then

$$
G(z)=\frac{1}{2 \pi} \int_{0}^{2 \pi} \frac{e^{i \vartheta}+z}{e^{i \vartheta}-z} \mu(\vartheta) d \vartheta-\mu_{0}, \quad \text { where } \mu_{0}=\frac{1}{2 \pi} \int_{0}^{2 \pi} \mu(\vartheta) d \vartheta
$$

Thus instead of (1.1) we may study the equivalent representations (1.3) or (1.5). This leads us to our first result.

Th e o r e m 1. For $g(z)$, given by (1.1), to have a representation (1.2), where $\mu(\vartheta)$ is measurable and $|\mu(\vartheta)| \leq M$, the following conditions are necessary and sufficient.

(i) The integral

$$
I\left(r, \varphi_{1}, \varphi_{2}\right)=\int_{\varphi_{1}}^{\varphi_{2}} u\left(r e^{i t}\right) d t
$$

satisfies $\left|I\left(r, \varphi_{1}, \varphi_{2}\right)\right| \leq 2 M, \quad 0<r<1, \quad-\infty<\varphi_{1}<\varphi_{2}<+\infty$.

(ii) If the real constants $\varphi_{1}$ and $\mu\left(\varphi_{1}\right)$ are suitably chosen

$$
I\left(r, \varphi_{1}, \varphi_{2}\right) \rightarrow \mu\left(\varphi_{2}\right)-\mu\left(\varphi_{1}\right) \quad \text { as } r \rightarrow 1
$$

for almost all fixed $\varphi_{2}$, where $\left|\mu\left(\varphi_{j}\right)\right| \leq M, j=1,2$.

Since Theorem 1 is very simple we give the proof now. Suppose in fact that $g(z)$ possesses the representation (1.2) and so $G(z)$ possesses the representation (1.5) where $|\mu(\vartheta)| \leq M$. Taking real parts we deduce that

$$
U\left(\varrho e^{i \varphi}\right)=\frac{1}{2 \pi} \int_{0}^{2 \pi} \frac{\left(1-\varrho^{2}\right) \mu(\vartheta) d \vartheta}{1-2 \varrho \cos (\vartheta-\varphi)+\varrho^{2}}-\mu_{0} .
$$

Thus

$$
\left|U\left(\varrho e^{i \varphi}\right)+\mu_{0}\right| \leq M \frac{1}{2 \pi} \int_{0}^{2 \pi} \frac{\left(1-\varrho^{2}\right) d \vartheta}{1-2 \varrho \cos (\vartheta-\varphi)+\varrho^{2}}=M .
$$

Hence for real $\varphi_{1}$ and $\varphi_{2}$ and $0<r<1$, we have

$$
I\left(r, \varphi_{1}, \varphi_{2}\right)=\left|\left(U\left(r e^{i \varphi_{2}}\right)+\mu_{0}\right)-\left(U\left(r e^{i \varphi_{1}}\right)+\mu_{0}\right)\right| \leq 2 M .
$$

Next it follows that whenever $\mu(\varphi)$ is the derivative of its integral and in particular for almost all $\varphi[2$, Theorem 1.2, p. 4]

$$
U\left(\varrho e^{i \varphi}\right) \rightarrow \mu(\varphi)-\mu_{0}, \quad \text { as } \varrho \rightarrow 1 .
$$


We choose for $\varphi_{1}$ such a value of $\varphi$ and deduce that for almost all $\varphi_{2}$

$$
I\left(r, \varphi_{1}, \varphi_{2}\right)=U\left(r e^{i \varphi_{2}}\right)-U\left(r e^{i \varphi_{1}}\right) \rightarrow \mu\left(\varphi_{2}\right)-\mu\left(\varphi_{1}\right) .
$$

Thus (i) and (ii) are necessary.

Suppose next that (i) and (ii) are satisfied. Then Poisson's formula gives for $0<r<\varrho<1$

$$
U\left(r e^{i \vartheta}\right)=\frac{1}{2 \pi} \int_{\varphi_{1}}^{\varphi_{1}+2 \pi} \frac{\varrho^{2}-r^{2}}{\varrho^{2}-2 r \varrho \cos (\varphi-\vartheta)+r^{2}} U\left(\varrho e^{i \varphi}\right) d \varphi .
$$

We have

$$
U\left(\varrho e^{i \varphi_{2}}\right)-U\left(\varrho e^{i \varphi_{1}}\right)=I\left(\varrho, \varphi_{1}, \varphi_{2}\right) .
$$

Thus

$$
U\left(r e^{i \vartheta}\right)=\frac{1}{2 \pi} \int_{\varphi_{1}}^{\varphi_{1}+2 \pi} \frac{\varrho^{2}-r^{2}}{\varrho^{2}-2 r \varrho \cos (\vartheta-\varphi)+r^{2}} I\left(\varrho, \varphi_{1}, \varphi\right) d \varphi+U\left(\varrho e^{i \varphi_{1}}\right) \text {. }
$$

We take $r, \vartheta$ fixed and let $\varrho$ tend to one through the sequence $\varrho_{n}=$ $1-1 / n$.

Then it follows from (i) and (ii) that the integrand converges boundedly to

$$
\frac{1-r^{2}}{1-2 r \cos (\vartheta-\varphi)+r^{2}}\left(\mu(\varphi)-\mu\left(\varphi_{1}\right)\right)
$$

so that the integral converges to

$$
\frac{1}{2 \pi} \int_{\varphi_{1}}^{\varphi_{1}+2 \pi} \frac{1-r^{2}}{1-2 r \cos (\vartheta-\varphi)+r^{2}}\left(\mu(\varphi)-\mu\left(\varphi_{1}\right)\right) d \varphi .
$$

Thus also $U\left(\varrho e^{i \varphi_{1}}\right)$ must tend to a limit $c$, since the left hand side is fixed. We define the function $\mu(\varphi)$ by periodicity outside $\left[\varphi_{1}, \varphi_{1}+2 \pi\right)$. Then

$$
\begin{aligned}
U\left(r e^{i \vartheta}\right) & =\frac{1}{2 \pi} \int_{\varphi_{1}}^{\varphi_{1}+2 \pi} \frac{1-r^{2}}{1-2 r \cos (\vartheta-\varphi)+r^{2}}\left(\mu(\varphi)-\mu\left(\varphi_{1}\right)\right) d \varphi+c \\
& =\frac{1}{2 \pi} \int_{\varphi_{1}}^{\varphi_{1}+2 \pi} \frac{1-r^{2}}{1-2 r \cos (\vartheta-\varphi)+r^{2}} \mu(\varphi) d \varphi+c_{1} \\
& =\frac{1}{2 \pi} \int_{0}^{2 \pi} \frac{\left(1-r^{2}\right) \mu(\varphi) d \varphi}{1-2 r \cos (\vartheta-\varphi)+r^{2}}+c_{1} .
\end{aligned}
$$


Setting $r=0$, we deduce that

$$
c_{1}=\frac{-1}{2 \pi} \int_{0}^{2 \pi} \mu(\varphi) d \varphi=-\mu_{0} .
$$

This leads in turn to (1.5), (1.3) and (1.2) and completes the proof of Theorem 1 .

1.1. In a previous paper the second author [4] showed that the representation (1.2) is certainly valid if

$$
u\left(r e^{i \vartheta}\right)<c \log \frac{1}{1-r}, \quad 0<r<1
$$

where $c$ is a constant. He also obtained conditions on the function $\mu(\vartheta)$, which were necessary and sufficient for (1.7) to hold for some constant $c$. In particular he showed that in this case $\mu(\vartheta)$ has left and right limits everywhere, so that (1.7) holds for all $\varphi_{1}$ and $\varphi_{2}$. Our main aim in this paper is to consider to what extent the condition (1.7) can be weakened while still allowing a representation of the form (1.2) with a bounded $\mu$.

We prove

Th e o r e $\mathrm{m}$ 2. Suppose that $k(r)$ is a positive continuous nondecreasing function of $r$ in $0 \leq r<1$ and

$$
u\left(r e^{i \vartheta}\right) \leq k(r), \quad 0 \leq r<1,0 \leq \vartheta \leq 2 \pi,
$$

where $u(z)$ is defined by (1.1). Then if

$$
J=\int_{0}^{1} \sqrt{\frac{k(r)}{1-r}} d r
$$

is finite, the function $g(z)$ possesses a representation (1.2) with $|\mu(\vartheta)| \leq$ $A_{0} J^{2}$, where $A_{0}$ is an absolute constant. Further (1.6) holds for all real $\varphi_{1}, \varphi_{2} \cdot$

The following consequence of (1.2) is almost trivial.

The or e m 3. If $g(z)$ is given by (1.2) with $|\mu(\vartheta)| \leq M$, then

$$
\left|g\left(r e^{i \varphi}\right)\right| \leq \frac{2 M r}{1-r^{2}}, \quad 0<r<1, \quad 0 \leq \varphi \leq 2 \pi .
$$

In fact we deduce from (1.3) that 


$$
\begin{aligned}
\left|g\left(r e^{i \varphi}\right)\right| & \leq 2 M r \frac{1}{2 \pi} \int_{0}^{2 \pi} \frac{d \vartheta}{\left|e^{i \vartheta}-r e^{i \varphi}\right|^{2}} \\
& =\frac{2 M r}{1-r^{2}} \frac{1}{2 \pi} \int_{0}^{2 \pi} \frac{\left(1-r^{2}\right) d \vartheta}{1-2 r \cos (\vartheta-\varphi)+r^{2}}=\frac{2 M r}{1-r^{2}},
\end{aligned}
$$

by Poisson's formula. Thus we deduce that (1.8) leads to (1.10) with $M=A_{0} J^{2}$. With some subsidiary monotonicity conditions on $k(r)$ a slightly less precise result of this type was proved by Linden [6], who also gave some examples in the opposite direction.

All the above results break down when $J$ is infinite. We have

$\mathrm{T}$ h e o r e m 4. Suppose that $k(r)$ is as in Theorem 2, but that $J=+\infty$. Then there exists $g=u+i v$ given by (1.1), satisfying (1.8) but such that

$$
(1-r) u(r) \rightarrow-\infty, \quad \text { as } r \rightarrow 1
$$

and

(1.12) $I(r, 0, \varphi)$

$$
=\frac{1}{2 \pi} \int_{0}^{\varphi} u\left(r e^{i t}\right) d t \rightarrow+\infty, \quad \text { as } r \rightarrow 1, \text { for } 0<\varphi<2 \pi .
$$

Thus $g$ cannot possess a representation (1.2) with bounded $\mu(\vartheta)$.

Suppose that there exists a function $z=\varphi(w)$, which is regular in $|w|<1$, real and increasing on the segment $[0,1]$ of the real axis and that $\varphi(0)=r_{0} \geq 0, \varphi(1)=1$ and

$$
|\varphi(w)|<1, \text { for }|w|<1 \text {. }
$$

Suppose further that

$$
|1-\varphi(w)|<C_{1}|1-w|, \quad 0 \leq w<1
$$

where $C_{1}$ is a constant.

Suppose next that $f(z)$ is meromorphic in $|z|<1$ and that

$$
T\left(r, f\left\{e^{i \vartheta} \varphi(w)\right\}\right) \leq C_{2}, \quad 0 \leq \vartheta \leq 2 \pi, 0<r<1,
$$

where $T(r, \psi)$ denotes the Nevanlinna characteristic of $\psi(w)$ in $|w|<r$, and $C_{2}$ is a positive constant. Then we say that $f$ has locally bounded characteristic (1.b.c.) in $|z|<1$.

Our positive results will follow from

$\mathrm{Th}$ e o r e m 5. If $f(z)$ is regular in $|z|<1$ and

$$
\log \left|f\left(r e^{i \vartheta}\right)\right| \leq k(r)
$$


there, where $k(r)$ satisfies the hypotheses of Theorem 2, then we can find $\varphi(w)$ such that (1.13) and (1.14) are satisfied with $r_{0}=1 / e, C_{1}$ and $C_{2} / J^{2}$ absolute constants.

The o r e m 6. If $g$ is given by (1.1) and $f=e^{g}$ satisfies (1.14) subject to (1.13) then $g$ satisfies (1.2) with $|\mu(\vartheta)| \leq C_{2} C_{3}$ where $C_{3}$ depends on $C_{1}$ and $r_{0}$ only. Further (1.6) holds for all real $\varphi_{1}, \varphi_{2}$.

Theorem 6 shows that if $f=e^{g}$ has 1.b.c., then $g$ possesses a representation (1.2). Thus we obtain more general classes of such functions, than merely those satisfying (1.8). For instance if $f$ is a normal non-zero function in the sense of Lehto and Virtanen [5], or alternately if $f$ is regular, $f \neq 0, f^{(p)}(z) \neq 1$, then these conditions are satisfied. In the above cases we may take $\varphi(w)=\frac{1}{4}+\frac{3}{4} w$ (see e.g. [3]).

The remainder of the paper will proceed as follows. In sections 2, 3 and 4 we construct a function in terms of conformal mappings which yields the example whose existence is asserted in Theorem 4. Section 5 is devoted to the proof of Theorem 5, which is also based on conformal mappings. Both these results use the first and second fundamental inequalities of Ahlfors [1]. In section 6 we prove Theorem 6 , which is relatively elementary and Theorem 2, which is an immediate consequence of Theorems 5 and 6 .

2. The Conformal Mappings. Most of our proofs depend on conformal maps of various striplike domains onto strips. All the results we require can be deduced from the fundamental first and second inequalities of Ahlfors [1]. We shall consider a domain $\Delta$ in the $\zeta=\xi+i \eta$ plane given by

$$
|\eta|<\frac{1}{2} \vartheta(\xi), \quad-\infty<\xi<+\infty
$$

where we shall assume that $\vartheta(\xi)$ is lower semi-continuous,

$$
\frac{\pi}{2} \leq \vartheta(\xi) \leq \frac{3}{2} \pi,
$$

and that $\vartheta(\xi)$ has finite variation $V\left(\xi_{1}, \xi_{2}\right)$ in any finite interval $\left[\xi_{1}, \xi_{2}\right]$.

We consider the map $s=s(\zeta)$ of $\Delta$ onto the strip $S$ given by $|\tau|<\pi / 2$ in the $s=\sigma+i \tau$ plane, which is symmetrical, i.e. $\sigma=\mp \infty$ correspond to $\xi=\mp \infty$ and further $s(0)=0$.

Then the two basic inequalities of Ahlfors [1] can be written as follows

$\mathrm{L} \mathrm{e} \mathrm{m} \mathrm{m} \mathrm{a} 1$. If $s_{j}=\sigma_{j}+i \tau_{j}$ correspond to $\zeta_{j}=\xi_{j}+i \eta_{j}, j=1,2$, where $\xi_{2}-\xi_{1} \geq 3 \pi$ then

$$
\sigma_{2}-\sigma_{1} \geq \pi \int_{\xi_{1}}^{\xi_{2}} \frac{d t}{\vartheta(t)}-4 \pi
$$


and

$$
\sigma_{2}-\sigma_{1}<\pi \int_{\xi_{1}}^{\xi_{2}} \frac{d t}{\vartheta(t)}+A_{1}\left\{1+V\left(\xi_{1}-6 \pi, \xi_{2}+6 \pi\right)\right\},
$$

where $A_{1}=2000$ and $V\left(x_{1}, x_{2}\right)$ is the variation of $\vartheta(x)$ in $\left[x_{1}, x_{2}\right]$.

The first inequality of Ahlfors [1, p. 10], states that (2.3) holds if

$$
\int_{\xi_{1}}^{\xi_{2}} \frac{d t}{\vartheta(t)} \geq 2
$$

which is certainly true for $\xi_{2}-\xi_{1} \geq 3 \pi$, in view of (2.2). Similarly Ahlfors's second inequality [1, p. 16] states that

$$
\sigma_{2}-\sigma_{1}<\pi \int_{\xi_{1}}^{\xi_{2}} \frac{d t}{\vartheta(t)}+8 \pi+16 \pi L^{2} \frac{l^{2}+V_{1}}{l^{4}} .
$$

Here $l, L$ can be taken to be the lower and upper bounds of $\vartheta(x)$ for $-\infty<x<+\infty$ and $V_{1}$ is the variation of $\vartheta(x)^{2}$ in $\left[\xi_{1}-4 L, \xi_{2}+4 L\right]$. In our case we may set $l=\pi / 2, L=3 \pi / 2$, and

$$
V_{1}=\int 2 \vartheta(x)|d \vartheta(x)| \leq 3 \pi \int|d \vartheta(x)|=3 \pi V
$$

say. Thus

$$
\begin{aligned}
\sigma_{2}-\sigma_{1} & <\pi \int_{\xi_{1}}^{\xi_{2}} \frac{d x}{\vartheta(x)}+8 \pi+16 \pi \cdot \frac{9}{4} \pi^{2} \cdot \frac{(\pi / 2)^{2}+3 \pi V}{(\pi / 2)^{4}} \\
& =\pi \int_{\xi_{1}}^{\xi_{2}} \frac{d x}{\vartheta(x)}+152 \pi+1728 \mathrm{~V} .
\end{aligned}
$$

This proves (2.4).

2.1. While Lemma 1 is used for the proof of Theorems 4 and 5 , the results that follow will be used specifically for the proof of Theorem 4 . We denote by $A$ a positive absolute constant, not necessarily the same each time. Specific constants will be denoted by $A_{2}, A_{3}, \ldots$.

We now suppose that $\pi \leq \vartheta(x)<3 \pi / 2$ and write

$$
\vartheta(x)=\pi+\varepsilon(x), \quad 0 \leq \varepsilon(x)<\pi / 2 .
$$

We also assume that $\varepsilon(x)=0$ for $x<0$, 
(2.5) $\varepsilon(x)=\varepsilon_{n}=$ constant for $(n-1) A_{2}<x<n A_{2}, \quad n \geq 1$, and $\varepsilon(x)=\min \left\{\varepsilon_{n}, \varepsilon_{n+1}\right\}$ for $x=n A_{2}$. Here $A_{2}=8 \pi A_{1}$.

$\mathrm{L} \mathrm{e} \mathrm{m} \mathrm{m}$ a 2 . With the above hypotheses if further

$$
I(x)=\int_{0}^{x} \varepsilon(t) d t \rightarrow+\infty, \quad \text { as } x \rightarrow+\infty
$$

then for $\xi_{2} \geq 3 \pi$

$$
\sigma_{2} \leq \xi_{2}-\frac{1}{4 \pi} I\left(\xi_{2}\right)+A_{2}
$$

and so

$$
\sigma_{2}-\xi_{2} \rightarrow-\infty, \text { as } \xi_{2} \rightarrow+\infty \text {. }
$$

We suppose that $(n-1) A_{2} \leq \xi_{2} \leq n A_{2}$, and set $\zeta_{1}=0$, so that $\sigma_{1}=0$. Then

$$
V=V\left(-6 \pi, \xi_{2}+6 \pi\right) \leq 2 \sum_{0}^{n+1} \varepsilon_{\nu}
$$

On the other hand

$$
\frac{1}{\vartheta(t)}=\frac{1}{\pi+\varepsilon(t)} \leq \frac{1}{\pi}-\frac{\varepsilon(t)}{2 \pi^{2}}
$$

Thus

$$
\pi \int_{0}^{\xi_{2}} \frac{d t}{\vartheta(t)} \leq \xi_{2}-\frac{1}{2 \pi} I\left(\xi_{2}\right)
$$

Using (2.5) we see that

$$
I\left(\xi_{2}\right) \geq \sum_{1}^{n-1} A_{2} \varepsilon_{v} \geq \frac{A_{2}}{2}\left(V-2 \varepsilon_{0}-2 \varepsilon_{n}-2 \varepsilon_{n+1}\right) \geq \frac{A_{2}}{2}(V-3 \pi) ;
$$

i.e.

$$
V \leq \frac{2}{A_{2}} I\left(\xi_{2}\right)+3 \pi
$$

Thus (2.4) yields

$$
\begin{aligned}
\sigma_{2} & \leq \xi_{2}-\frac{1}{2 \pi} I\left(\xi_{2}\right)+A_{1}\left(1+\frac{2}{A_{2}} I\left(\xi_{2}\right)+3 \pi\right) \\
& \leq \xi_{2}-\frac{1}{4 \pi} I\left(\xi_{2}\right)+A_{2} .
\end{aligned}
$$


This proves (2.7) and hence (2.8) and completes the proof of Lemma 2. Having obtained some estimates for $\sigma$, we next turn to estimates for $\tau$. Let $S^{+}$be the upper half of $S$ i.e. the strip

$$
s=\sigma+i \tau, \quad-\infty<\sigma<+\infty, 0<\tau<\frac{\pi}{2} .
$$

$\mathrm{L}$ e m m a 3. Suppose that $(n-1) A_{2} \leq \xi \leq n A_{2}$. Let $\varepsilon_{n}^{\prime}=$ $\min \left(\varepsilon_{n}, \varepsilon_{n-1}, \varepsilon_{n+1}\right)$ and $\varepsilon_{n}^{\prime \prime}=\max \left(\varepsilon_{n}, \varepsilon_{n-1}, \varepsilon_{n+1}\right)$. If $s=\sigma+i \tau$ corresponds to $\xi+i \eta$, then we have

$$
\frac{\pi}{4}+\frac{1}{8} \varepsilon_{n}^{\prime}<\eta(s)<\frac{3 \pi}{8}, \quad \text { if } \tau=\frac{\pi}{4} .
$$

Further if $\eta>\pi / 2$, we have

$$
\tau>\frac{\pi}{2}-\varepsilon_{n}^{\prime \prime}
$$

For $-\infty<s_{0}<\infty, s \in S^{+}$, we write

$$
h\left(s, s_{0}\right)=\frac{-1}{\pi} \tan ^{-1}\left(\frac{e^{2 \sigma} \cos (2 \tau)+e^{2 s_{0}}}{e^{2 \sigma} \sin (2 \tau)}\right)+\frac{1}{2} .
$$

Then $h\left(s, s_{0}\right)$ is harmonic and bounded in $S^{+}$. Also $h\left(s, s_{0}\right)$ has boundary values 0 for $\tau=0$, and $\tau=\pi / 2, \sigma<s_{0}$ and boundary values one for $\tau=\pi / 2, \sigma>s_{0}$. Suppose that $s=s_{1}+i \pi / 2, s_{2}+i \pi / 2$ correspond to

$$
\zeta=(n-2) A_{2}+\frac{1}{2} i \vartheta\left((n-2) A_{2}\right), \quad(n+1) A_{2}+\frac{1}{2} i \vartheta\left((n+1) A_{2}\right)
$$

respectively. Then it follows from (2.3) that

$$
s_{2}-\sigma \geq \frac{1}{2} A_{2}-4 \pi, \quad \sigma-s_{1} \geq \frac{1}{2} A_{2}-4 \pi .
$$

Consider now

$$
\begin{gathered}
h_{1}(s)=\tau+\frac{1}{2} \varepsilon_{n}^{\prime}\left\{h\left(s, s_{1}\right)-h\left(s, s_{2}\right)\right\}, \\
h_{2}(s)=\frac{3}{2} \tau-\left(\frac{\pi}{4}-\frac{1}{2} \varepsilon_{n}^{\prime \prime}\right)\left\{h\left(s, s_{1}\right)-h\left(s, s_{2}\right)\right\} .
\end{gathered}
$$

We note that $h_{1}(s), h_{2}(s)$ have boundary values zero for $\tau=0$. For $\tau=\pi / 2$ and $\sigma<s_{1}$ or $\sigma>s_{2}, h_{1}(s)=\pi / 2, h_{2}(s)=3 \pi / 4$. 
Finally for $s_{1}<\sigma<s_{2}, \tau=\pi / 2, h_{1}(s)=(1 / 2)\left(\pi+\varepsilon_{n}^{\prime}\right)$, and $h_{2}(s)=$ $(1 / 2)\left(\pi+\varepsilon_{n}^{\prime \prime}\right)$. Thus we deduce from the maximum principle and $(2.5)$ that

(2.12) $h_{1}(s)<\eta(s)<h_{2}(s)<\frac{3}{2} \tau, \quad-\infty<\sigma<+\infty, 0<\tau<\frac{1}{2} \pi$.

Suppose now first that $\tau=\pi / 4$. Then

$$
h\left(s, s_{2}\right)=\frac{1}{2}-\frac{1}{\pi} \tan ^{-1}\left(e^{2 s_{2}-2 \sigma}\right)=\frac{1}{\pi} \tan ^{-1}\left(e^{2 \sigma-2 s_{2}}\right)<\frac{1}{\pi} e^{2 \sigma-2 s_{2}}<\frac{1}{8}
$$

in view of (2.11).

Similarly

$$
h\left(s, s_{1}\right)>\frac{1}{2}-\frac{1}{\pi} e^{2 s_{1}-2 \sigma}>\frac{3}{8} .
$$

Thus

$$
\eta(s)>h_{1}(s)>\tau+\frac{1}{2} \varepsilon_{n}^{\prime}\left(\frac{3}{8}-\frac{1}{8}\right)=\frac{\pi}{4}+\frac{1}{8} \varepsilon_{n}^{\prime} .
$$

Since also $\eta(s)<3 \tau / 2$, we deduce $(2.9)$.

Next suppose that $\eta>\pi / 2$. Then we deduce that $\tau>\pi / 4$. Also

$$
\begin{aligned}
h\left(s, s_{2}\right) & =\frac{1}{\pi} \tan ^{-1}\left(\frac{\sin (2 \tau)}{\varepsilon^{2 s_{2}-2 \sigma}+\cos (2 \tau)}\right)<\frac{1}{\pi} \tan ^{-1}\left\{\frac{\pi-2 \tau}{e^{2 s_{2}-2 \sigma}-1}\right\} \\
& <\frac{1}{8}(\pi-2 \tau)
\end{aligned}
$$

in view of (2.11). Similarly

$$
h\left(s, s_{1}\right)=\frac{2}{\pi} \tau-\frac{1}{\pi} \tan ^{-1}\left\{\frac{\sin (2 \tau)}{e^{2 \sigma-2 s_{1}}+\cos (2 \tau)}\right\}>\frac{2 \tau}{\pi}-\frac{1}{8}(\pi-2 \tau) .
$$

Thus

$$
\begin{aligned}
h_{2}(s) & <\frac{3}{2} \tau-\left(\frac{\pi}{4}-\frac{1}{2} \varepsilon_{n}^{\prime \prime}\right)\left(\frac{2}{\pi} \tau-\frac{1}{4}(\pi-2 \tau)\right) \\
& <\left(1+\frac{1}{\pi} \varepsilon_{n}^{\prime \prime}\right) \tau+\frac{1}{4}(\pi-2 \tau) .
\end{aligned}
$$

Thus if $\eta(s)>\pi / 2$, we deduce from (2.12) that

$$
\pi<2 \tau\left(1+\frac{1}{\pi} \varepsilon_{n}^{\prime \prime}\right)+\frac{1}{2}(\pi-2 \tau),
$$




$$
\frac{1}{2}(\pi-2 \tau)<\frac{2 \tau}{\pi} \varepsilon_{n}^{\prime \prime}<\varepsilon_{n}^{\prime \prime}, \quad \text { i.e. } \tau>\frac{\pi}{2}-\varepsilon_{n}^{\prime \prime} .
$$

This proves (2.10) and completes the proof of Lemma 3.

3. The construction of $\varepsilon(x)$ in terms of $k(r)$. It is inconvenient to work with the quantities $\varepsilon_{n}^{\prime}, \varepsilon_{n}^{\prime \prime}$, and we wish to obtain results in terms of $\varepsilon_{n}$. In order to do this we now make the weak monotonicity assumption

$$
\varepsilon_{n+1} \geq A_{3} \varepsilon_{n}, \quad n=0,1,2, \ldots
$$

where $0<A_{3}<1$. We continue to suppose that $\varepsilon(x)$ is defined as above and in particular that (2.6) holds. Evidently (2.6) is equivalent to

$$
\sum_{1}^{\infty} \varepsilon_{n}=+\infty
$$

We now set

$$
\begin{gathered}
s=\frac{1}{2}\left\{\log \frac{1+z}{1-z}+i \frac{\pi}{2}\right\}, \\
w=i e^{2 \zeta}=u+i v
\end{gathered}
$$

and write $w=f(z),|z|<1$. We shall show that if the $\varepsilon_{n}$ are suitably defined the function $f(z)$, slightly renormalized, will satisfy the conclusions for $g(z)$ in Theorem 4. The next Lemma shows that (1.11) and (1.12) certainly hold.

$\mathrm{L}$ e $\mathrm{m} \mathrm{m} \mathrm{a} \mathrm{4.} \mathrm{If} w=u+i v=f(r)$ is defined by (3.3) and (3.4) on the positive real axis then

$$
(1-r) u(r) \rightarrow-\infty, \quad \text { as } r \rightarrow 1
$$

and

$$
\int_{1 / 2}^{1} v(r) \frac{d r}{r}=-\infty
$$

Thus also

$$
\int_{0}^{\vartheta} u\left(r e^{i t}\right) d t \rightarrow+\infty, \text { as } r \rightarrow 1 \text { for } 0<\vartheta<2 \pi .
$$

We have for $0<r<1$

$$
s=\sigma+i \tau=\frac{1}{2} \log \frac{1+r}{1-r}+i \frac{\pi}{4},
$$




$$
\begin{aligned}
u+i v & =\exp \left\{2 \xi+i\left(2 \eta+\frac{\pi}{2}\right)\right\} \\
& =e^{2 \xi}(-\sin (2 \eta)+i \cos (2 \eta)) .
\end{aligned}
$$

Since $\tau=\pi / 4$, we deduce from (2.9) that

$$
u \leq-\frac{1}{\sqrt{2}} e^{2 \xi} .
$$

Again (2.7) shows that

$$
\xi-\sigma \rightarrow+\infty, \text { as } \sigma \rightarrow+\infty .
$$

Using (3.8) to (3.10) we deduce that

$$
\log \{(1-r)(-u)\} \rightarrow+\infty, \quad \text { as } r \rightarrow 1,
$$

and this proves (3.5).

Next we deduce from (2.9) and (3.1) that for $s=\sigma+i \pi / 4$,

$$
\eta>\frac{\pi}{4}+\frac{1}{8} \varepsilon_{n}^{\prime}>\frac{\pi}{4}+\frac{1}{8} A_{3}^{2} \varepsilon_{n-1},
$$

and so

(3.11) $v=e^{2 \xi} \cos (2 \eta)<-e^{2 \xi} \sin \left(\frac{A_{3}^{2}}{4} \varepsilon_{n-1}\right)<-\frac{A_{3}^{2} \varepsilon_{n-1}}{2 \pi} e^{2 \xi}$.

We now write $\xi_{n}=n A_{2}$, and suppose that $\sigma=\sigma_{n}, r=r_{n}$, correspond to $\xi=\xi_{n}$ on the real $r$ axis, where $s, \xi, r$ are related by (3.8) and (3.9). Then Lemma 1, (2.3) and (2.2) show that

$$
\sigma_{n+1}-\sigma_{n}>\frac{2}{3} A_{2}-4 \pi>1
$$

Hence

$$
\begin{gathered}
\log \left(\frac{1+r_{n}}{1-r_{n}} \frac{1-r_{n+1}}{1+r_{n+1}}\right)<-2, \\
1-r_{n+1}<\frac{1}{2}\left(1-r_{n}\right) .
\end{gathered}
$$

In view of (3.11) we deduce that

$$
\int_{r_{n-1}}^{r_{n}}-v(r) \frac{d r}{r} \geq \frac{1}{2}\left(1-r_{n-1}\right) \inf _{(n-1) A_{2} \leq \xi \leq n A_{2}} \frac{A_{3}^{2} \varepsilon_{n-1}}{2 \pi} e^{2 \xi}
$$




$$
>\frac{A_{3}^{2} \varepsilon_{n-1}}{4 \pi}\left(1-r_{n-1}\right) e^{2 \xi_{n-1}}>\frac{A_{3}^{2} \varepsilon_{n-1}}{4 \pi} \exp \left(2 \xi_{n-1}-2 \sigma_{n-1}\right)>\varepsilon_{n-1}
$$

for $n>n_{0}$, in view of (2.8). Thus

$$
\int_{r_{n_{0}-1}}^{1}-v(r) \frac{d r}{r}>\sum_{n_{0}}^{\infty} \varepsilon_{n-1}=+\infty
$$

and this proves (3.6). To deduce (3.7) we note that for fixed $\vartheta, 0<\vartheta<2 \pi$, we have, as $r \rightarrow 1$,

$$
\begin{aligned}
\int_{0}^{\vartheta} u\left(r e^{i t}\right) d t & =\vartheta u(0)+\int_{0}^{r}\left\{v\left(\varrho e^{i \vartheta}\right)-v(\varrho)\right\} \frac{d t}{t} \\
& =-\int_{1 / 2}^{r} \frac{v(\varrho) d \varrho}{\varrho}+O(1) \rightarrow+\infty
\end{aligned}
$$

This proves (3.7) and completes the proof of Lemma 4.

3.1. To complete the proof of Theorem 4, we need to construct a function $\varepsilon(t)$ satisfying the above conditions and then obtain estimates for $u$ in terms of $\varepsilon(t)$. We proceed in a number of stages.

$\mathrm{L}$ e $\mathrm{m} \mathrm{m}$ a 5 . Let $k(r)$ be a nondecreasing continuous function of $r$ for $0 \leq r<1$, such that $k(0)=1$, and

$$
\int_{0}^{1} \sqrt{\frac{k(r)}{1-r}} d r=+\infty
$$

For $\xi \geq 0$, we define $\varepsilon_{1}(\xi)$ as follows. Let $r$ be the unique number such that

$$
e^{2 \xi}=\sqrt{\frac{k(r)}{1-r}}
$$

and set

$$
\varepsilon_{1}(\xi)=\sqrt{(1-r) k(r)} .
$$

Then $\varepsilon_{1}(t)$ is a positive function of $t$, such that for $0<t_{1}<t_{2}<\infty$ we have

$$
\varepsilon_{1}\left(t_{2}\right) \geq e^{2\left(t_{1}-t_{2}\right)} \varepsilon_{1}\left(t_{1}\right)
$$

Also 


$$
\int_{0}^{\infty} \varepsilon_{1}(\xi) d \xi=+\infty .
$$

By hypothesis $\{k(r) /(1-r)\}^{1 / 2}$ is a continuous strictly increasing function of $r$ in $[0,1)$ which is equal to 1 when $r=0$ and tends to $\infty$ as $r \rightarrow 1$. Thus (3.13) has a unique solution for $\xi \geq 0$, and $r$ is a continuous strictly increasing function of $\xi$. Thus $\varepsilon_{1}$ is a continuous function of $r$ in $[0,1)$, and so of $\xi$ in $[0, \infty)$, and $\varepsilon_{1}(0)=1$.

Next let $r=r_{1}, r_{2}$ correspond to $t=t_{1}, t_{2}$, where $t_{2}>t_{1}$. Thus

$$
e^{4\left(t_{2}-t_{1}\right)}=\frac{k\left(r_{2}\right)\left(1-r_{1}\right)}{k\left(r_{1}\right)\left(1-r_{2}\right)} \geq \frac{k\left(r_{1}\right)\left(1-r_{1}\right)}{k\left(r_{2}\right)\left(1-r_{2}\right)}=\frac{\varepsilon_{1}\left(t_{1}\right)^{2}}{\varepsilon_{1}\left(t_{2}\right)^{2}},
$$

since $k(r)$ increases with $r$. This proves (3.15).

Finally let $r=r_{n}$ correspond to $\xi=n$ by (3.13). Then

$$
\begin{aligned}
\int_{r_{n}}^{r_{n+1}} \sqrt{\frac{k(r)}{1-r}} d r & \leq\left(r_{n+1}-r_{n}\right) \sqrt{\frac{k\left(r_{n+1}\right)}{1-r_{n+1}}}=\left(r_{n+1}-r_{n}\right) e^{2(n+1)} \\
& <\left(1-r_{n}\right) e^{2} \sqrt{\frac{k\left(r_{n}\right)}{1-r_{n}}}=e^{2} \varepsilon_{1}(n)<e^{4} \int_{n}^{n+1} \varepsilon_{1}(t) d t
\end{aligned}
$$

in view of (3.15). Now (3.16) follows from (3.12) and the proof of Lemma 5 is complete.

We now set

$$
\varepsilon_{2}(t)=\min \left\{1, \varepsilon_{1}(t)\right\} .
$$

It is evident that (3.15) and hence (3.16) still holds with $\varepsilon_{2}(t)$ instead of $\varepsilon_{1}(t)$. Next we have

L e m m a 6. Suppose that $0<a<1$, and set

$$
\varepsilon_{3}(t)=a \varepsilon_{2}(t) /\left\{1+\int_{0}^{t} \varepsilon_{2}(\tau) d \tau\right\} .
$$

Then for $t_{1} \leq t_{2} \leq t_{1}+1$, we have

$$
\varepsilon_{3}\left(t_{2}\right) \geq \frac{1}{2} e^{-2} \varepsilon_{3}\left(t_{1}\right) .
$$

Further

$$
\int_{0}^{\infty} \varepsilon_{3}(t) d t=\infty
$$


and, for $t \geq 0$, we have

$$
\varepsilon_{3}(t) \exp \left\{\frac{2}{\pi} \int_{0}^{t} \varepsilon_{3}(\tau) d \tau\right\} \leq a \varepsilon_{2}(t) .
$$

We note that in view of (3.17) we have

$$
\int_{t}^{t+1} \varepsilon_{2}(\tau) d \tau \leq 1
$$

Thus

$$
\begin{aligned}
\frac{\varepsilon_{3}\left(t_{1}\right)}{\varepsilon_{3}\left(t_{2}\right)}=\frac{\varepsilon_{2}\left(t_{1}\right)}{\varepsilon_{2}\left(t_{2}\right)} \frac{1+\int_{0}^{t_{2}} \varepsilon_{2}(\tau) d \tau}{1+\int_{0}^{t_{1}} \varepsilon_{2}(\tau) d \tau} \leq \frac{\varepsilon_{2}\left(t_{1}\right)}{\varepsilon_{2}\left(t_{2}\right)} \frac{2+\int_{0}^{t_{1}} \varepsilon_{2}(\tau) d \tau}{1+\int_{0}^{t_{1}} \varepsilon_{2}(\tau) d \tau} \\
\leq 2 \frac{\varepsilon_{2}\left(t_{1}\right)}{\varepsilon_{2}\left(t_{2}\right)} \leq 2 e^{2}
\end{aligned}
$$

in view of (3.15). This proves (3.19).

Next we set

$$
f(t)=\log \left\{1+\int_{0}^{t} \varepsilon_{2}(\tau) d \tau\right\}, \quad \varepsilon_{3}(t)=a f^{\prime}(t) .
$$

Thus

$$
\int_{0}^{t} \varepsilon_{3}(\tau) d \tau=a f(t) \rightarrow \infty, \quad \text { as } t \rightarrow \infty .
$$

This proves (3.20). Again since $a<1$,

$$
\varepsilon_{3}(t) \exp \left\{\frac{2}{\pi} \int_{0}^{t} \varepsilon_{3}(\tau) d \tau\right\} \leq \varepsilon_{3}(t) \exp \left\{\int_{0}^{t} f^{\prime}(\tau) d \tau\right\}=a \varepsilon_{2}(t) .
$$

This proves (3.21) and completes the proof of Lemma 6 .

We now set $\varepsilon_{3}(t)=0$ for $t \leq 0$, define

$$
\varepsilon_{n}=\inf _{(n-2) A_{2}<t \leq(n-1) A_{2}} \varepsilon_{3}(t),
$$

and define $\varepsilon(x)$ by (2.5). 
4. Proof of Theorem 4. We can now prove

$\mathrm{L} \mathrm{e} \mathrm{m} \mathrm{m}$ a 7 . If $k(r)$ satisfies the hypotheses of Theorem $4, k(0)=1$ and $\varepsilon(t)$ is defined as above, where $a$ is a sufficiently small absolute constant, then the corresponding function $u(z)$ satisfies the conclusions (1.8), (1.11) and (1.12) of Theorem 4.

Suppose that $(n-1) A_{2}<x<n A_{2}$. Then it follows from (3.19) and $(3.22)$

$$
\varepsilon(x)=\varepsilon_{n} \geq A_{4} \varepsilon_{3}\left\{(n-2) A_{2}\right\} \geq A_{5} \varepsilon_{3}\left(x-2 A_{2}\right) .
$$

Thus (2.6) follows from (3.20). Similarly (3.1) follows from (3.19), so that the conclusions of Lemma 4 hold. Thus we have (1.11) and (1.12) in view of (3.5) and (3.7).

It remains to prove (1.8). We recall (3.3), i.e.

$$
s=\sigma+i \tau=\frac{1}{2}\left\{\log \left(\frac{1+r e^{i \vartheta}}{1-r e^{i \vartheta}}\right)+i \frac{\pi}{2}\right\},
$$

and that $s$ corresponds to $\zeta=\xi+i \eta$ in the map discussed in Lemmas 1 to 3 . Evidently $\tau>0$ and so

$$
0<\eta<\frac{1}{2} \vartheta(\xi)=\frac{1}{2}(\pi+\varepsilon(\xi)) \text {. }
$$

Suppose first that $\xi \leq A_{2}$. Then, by (3.22) and $(2.5), \varepsilon(\xi)=0$ and (3.9) shows that

$$
u=-e^{2 \xi} \sin (2 \eta) \leq 0<k(0),
$$

so that (1.8) holds in this case. More generally, if $u \leq 0,(1.8)$ is trivial.

Next suppose that $\xi>A_{2}, u>0$. In this case we proceed to show that if the constant $a$ is small enough we have

$$
u\left(r e^{i \vartheta}\right)<\varepsilon_{1}(\xi) e^{2 \xi}
$$

and

$$
1-r<\varepsilon_{1}(\xi) e^{-2 \xi} .
$$

These inequalities yield (1.8) immediately. In fact let $r=\varrho$ correspond to $\xi$ by (3.13). Then (3.13) and (3.14) yield

$$
\varepsilon_{1}(\xi) e^{2 \xi}=k(\varrho), \quad \varepsilon_{1}(\xi) e^{-2 \xi}=1-\varrho .
$$

Thus (4.4) shows that $\varrho<r$ and (4.3) that

$$
u\left(r e^{i \vartheta}\right)<k(\varrho) \leq k(r)
$$

which is (1.8). Thus we need only establish the inequalities (4.3) and (4.4). 
We recall that $\varepsilon(\xi)=0$ for $\xi \leq A_{2}$ and that $\varepsilon(\xi)$ is defined by (3.22) and (2.5) for $\xi>A_{2}$.

We suppose that $(n-1) A_{2}<\xi \leq n A_{2}, n>1$, so that (4.2) yields

$$
u\left(r e^{i \vartheta}\right)=-e^{2 \xi} \sin (2 \eta),
$$

where $0<2 \eta<\vartheta(\xi)=\pi+\varepsilon(\xi)$. If $2 \eta \leq \pi$, we have $u \leq 0$, so that (1.8) is trivial. If $2 \eta>\pi$, we have

$$
\begin{aligned}
-\sin (2 \eta) & =\sin (2 \eta-\pi)<\sin \varepsilon(\xi)<\varepsilon(\xi) \\
& =\varepsilon_{n}=\inf _{(n-2) A_{2} \leq t \leq(n-1) A_{2}} \varepsilon_{3}(t) .
\end{aligned}
$$

Thus (3.19) yields $\varepsilon(\xi)<A_{6} \varepsilon_{3}(\xi)$. Hence, using (3.17) and (3.18) we have

$$
u\left(r e^{i \vartheta}\right)<\varepsilon(\xi) e^{2 \xi}<A_{6} a \varepsilon_{2}(\xi) e^{2 \xi}<\varepsilon_{1}(\xi) e^{2 \xi}
$$

provided that

$$
a<A_{6}^{-1} .
$$

Thus if (4.5) holds we deduce (4.3).

It remains to prove (4.4). We deduce from (4.1) that

$$
r e^{i \vartheta}=\frac{i e^{2 s}+1}{i e^{2 s}-1}, \quad r^{2}=\frac{1-2 e^{2 \sigma} \sin (2 \tau)+e^{4 \sigma}}{1+2 e^{2 \sigma} \sin (2 \tau)+e^{4 \sigma}} .
$$

Thus

$$
1-r<1-r^{2}=\frac{4 e^{2 \sigma} \sin (2 \tau)}{1+2 e^{2 \sigma} \sin (2 \tau)+e^{4 \sigma}}<4 e^{-2 \sigma} \sin (2 \tau),
$$

since $0<\tau<\pi / 2$, so that $\sin (2 \tau)>0$.

In view of (3.9) and since $u>0$, we have $\eta>\pi / 2$. We apply Lemma $3,(2.1)$ and (3.1). These show that

$$
\sin (2 \tau)<\pi-2 \tau<2 \varepsilon_{n}^{\prime \prime}<A_{7} \varepsilon_{n+1} \leq A_{7} \varepsilon_{3}(\xi),
$$

in view of (3.22).

Next Lemma 1, (2.3) shows that, since $\xi \geq A_{2}>3 \pi$,

$$
\begin{aligned}
\sigma & >\pi \int_{0}^{\xi} \frac{d t}{\vartheta(t)}-4 \pi=\pi \int_{0}^{\xi} \frac{d t}{\pi+\varepsilon(t)}-4 \pi \\
& >\xi-\frac{1}{\pi} \int_{0}^{\xi} \varepsilon(t) d t-4 \pi .
\end{aligned}
$$

Thus 


$$
e^{-2 \sigma}<A_{8} e^{-2 \xi} \exp \left\{\frac{2}{\pi} \int_{0}^{\xi} \varepsilon(t) d t\right\} .
$$

It follows from (3.22) that

$$
\varepsilon(t) \leq \varepsilon_{3}\left(t-A_{2}\right) .
$$

Thus

$$
\int_{0}^{\xi} \varepsilon(t) d t \leq \int_{-A_{2}}^{\xi-A_{2}} \varepsilon_{3}(t) d t \leq \int_{0}^{\xi} \varepsilon_{3}(t) d t+A_{9},
$$

since $0 \leq \varepsilon(t)<1$. Thus (4.6) to (4.9) show that for $\xi \geq 3 \pi$

$$
\begin{aligned}
1-r & <A_{10} \varepsilon_{3}(\xi) e^{-2 \xi} \exp \left\{\frac{2}{\pi} \int_{0}^{\xi} \varepsilon_{3}(t) d t\right\} \\
& <A_{10} a \varepsilon_{2}(\xi) e^{-2 \xi} \leq A_{10} a \varepsilon_{1}(\xi) e^{-2 \xi},
\end{aligned}
$$

in view of $(3.21),(3.17)$. Hence if $a=\min \left(A_{10}^{-1}, A_{6}^{-1}\right)$, we deduce that (4.4) holds. We have already proved (4.3) subject to (4.5). Thus the proof of Lemma 7 is complete.

Suppose now that $f(0)=\alpha+i \beta$. If $\alpha \geq 0, g(z)=f(z)-f(0)$ will also satisfy (1.8), (1.11) and (1.12) and in addition (1.1). If $\alpha<0$, we set

$$
g(z)=\frac{f(z)-f(0)}{1+|\alpha|}, \text { so that } \mathbf{R}\{g(z)\}<\frac{|\alpha|+k(r)}{1+|\alpha|} \leq k(r),
$$

if $k(0)=1$. If $k(0) \neq 1$, we construct $g(z) / k(0)$ to satisfy Theorem 4 with $k(r) / k(0)$, instead of $k(r)$. Thus Theorem 4 is proved in all cases.

5. Proof of Theorem 5. In order to prove Theorem 5 we also use conformal mapping. We make the preliminary transformation

$$
Z=X+i Y=\log \frac{1}{z}, \quad z=e^{-Z},
$$

which makes the unit disk $|z|<1$, punctured at the origin correspond to the half plane

We set

$$
D_{1}: X>0 \text {. }
$$

$$
X_{n}=2^{-n}, \quad n \geq 0 .
$$

Let $Y_{n}$ be a nonincreasing sequence of positive numbers such that 


$$
Y_{0}=Y_{1}=1, \quad \sum_{2}^{\infty} \frac{X_{n}}{Y_{n}} \leq 1 .
$$

Let $D_{2}$ be the domain given by

$$
\begin{gathered}
|Y|<X, \quad X>1, \\
|Y|<Y_{n}, \quad X_{n+1}<X \leq X_{n} .
\end{gathered}
$$

We note that $D_{2} \subset D_{1}$. Thus $D_{2}$ corresponds by (5.1) to a domain $D_{0}$ in $|z|<1$. We proceed to consider the map of $D_{2}$ onto the strip $S$ of section 2. To do this we set

$$
\zeta=\log \frac{1}{Z}=\xi+i \eta
$$

and proceed to show that $D_{2}$ corresponds in the $\zeta$ plane to a domain $\triangle$ given by (2.1).

To see this we note that $D_{2}$ consists of all points $Z=e^{-\xi-i \eta}$ where

$$
|\eta|<\frac{1}{2} \vartheta(\xi), \quad-\infty<\xi<\infty
$$

and the function $\vartheta(\xi)$ is defined as follows

$$
\vartheta(\xi)=\frac{1}{2} \pi, \quad \xi \leq-\frac{1}{2} \log 2,
$$

$$
\frac{1}{2} \vartheta(\xi)=\sin ^{-1}\left(Y_{n} e^{\xi}\right)
$$

$$
\frac{1}{2} \log \left(\frac{1}{X_{n}^{2}+Y_{n}^{2}}\right) \leq \xi \leq \frac{1}{2} \log \left(\frac{1}{X_{n+1}^{2}+Y_{n}^{2}}\right), \quad n=0 \text { to } \infty,
$$$$
\frac{1}{2} \vartheta(\xi)=\cos ^{-1}\left(X_{n+1} e^{\xi}\right),
$$

$$
\frac{1}{2} \log \left(\frac{1}{X_{n+1}^{2}+Y_{n}^{2}}\right) \leq \xi \leq \frac{1}{2} \log \left(\frac{1}{X_{n+1}^{2}+Y_{n+1}^{2}}\right), \quad n=0 \text { to } \infty .
$$

In view of (5.2) we have $Y_{n} \geq X_{n}$, and so $\pi / 2 \leq \vartheta(\xi) \leq \pi$, which yields (2.2). Thus we may apply Lemma 1.

To do this we next consider the total variation $V$ of $\vartheta(\xi)$. We note that $\vartheta(\xi)<\pi$ and that $\vartheta(\xi)$ is monotonic in each of the ranges $(5.6)$ to (5.8). Also in the ranges (5.7) and (5.8) we have 


$$
\begin{aligned}
\cos \left\{\frac{1}{2} \vartheta(\xi)\right\} & =\sin \left\{\frac{\pi}{2}-\frac{1}{2} \vartheta(\xi)\right\} \\
& \leq \max \left(\frac{X_{n}}{\sqrt{X_{n}^{2}+Y_{n}^{2}}}, \frac{X_{n+1}}{\sqrt{X_{n+1}^{2}+Y_{n+1}^{2}}}\right)
\end{aligned}
$$

Thus

$$
\pi-\vartheta(\xi) \leq \pi \max \left\{\frac{X_{n}}{Y_{n}}, \frac{X_{n+1}}{Y_{n+1}}\right\} .
$$

Thus the variation of $\vartheta(\xi)$ in the union of the two ranges (5.7) and (5.8) is at most

$$
2 \pi\left\{\frac{X_{n}}{Y_{n}}+\frac{X_{n+1}}{Y_{n+1}}\right\} .
$$

Thus the total variation $V$ of $\vartheta(\xi)$ in $-\infty<\xi<+\infty$ satisfies

$$
V \leq 2 \pi\left\{\sum_{n=0}^{\infty} \frac{X_{n}}{Y_{n}}+\sum_{n=1}^{\infty} \frac{X_{n}}{Y_{n}}\right\}=2 \pi\left(X_{0}+2 X_{1}+2 \sum_{2}^{\infty} \frac{X_{n}}{Y_{n}}\right) \leq 8 \pi
$$

in view of (5.2). Thus Lemma 1 is applicable with $V \leq 8 \pi$.

We now write

$$
\vartheta(\xi)=\pi-\varepsilon(\xi)
$$

Then (5.9) yields

$$
\varepsilon(\xi) \leq \pi \max \left\{\frac{X_{n}}{Y_{n}}, \frac{X_{n+1}}{Y_{n+1}}\right\} \quad \text { and } \quad \varepsilon(\xi) \leq \frac{\pi}{2},
$$

since $\vartheta(\xi) \geq \pi / 2$. Further if

$$
\xi_{n}=\frac{1}{2} \log \left(\frac{1}{X_{n}^{2}+Y_{n}^{2}}\right), \quad \xi_{n}^{\prime}=\frac{1}{2} \log \left(\frac{1}{X_{n+1}^{2}+Y_{n}^{2}}\right), \quad n \geq 1
$$

we have

$$
\int_{\xi_{n}}^{\xi_{n}^{\prime}} \varepsilon(\xi) d \xi \leq\left(\xi_{n}^{\prime}-\xi_{n}\right) \varepsilon\left(\xi_{n}\right) \leq\left(\log \frac{X_{n}}{X_{n+1}}\right) \varepsilon\left(\xi_{n}\right) \leq \pi \frac{X_{n}}{Y_{n}} \log 2 .
$$

Further for $\xi_{n}^{\prime} \leq \xi \leq \xi_{n+1}$, we have

$$
\frac{1}{2} \varepsilon(\xi)=\sin ^{-1}\left(X_{n+1} e^{\xi}\right), \quad \varepsilon(\xi) \leq \pi X_{n+1} e^{\xi},
$$




$$
\int_{\xi_{n}^{\prime}}^{\xi_{n+1}} \varepsilon(\xi) d \xi \leq \pi X_{n+1} e^{\xi_{n+1}} \leq \frac{\pi X_{n+1}}{Y_{n+1}}
$$

Also $\xi_{1} \leq 0$ in view of (5.2). Thus

$$
\int_{0}^{\infty} \varepsilon(\xi) d \xi \leq \pi(\log 2+1) \sum_{1}^{\infty} \frac{X_{n}}{Y_{n}} \leq \frac{3}{2} \pi(\log 2+1),
$$

in view of (5.2). Also for $\xi>0$ we deduce, using (5.11),

$$
\begin{aligned}
\pi \int_{0}^{\xi} \frac{d t}{\vartheta(t)} & =\pi \int_{0}^{\xi} \frac{d t}{\pi-\varepsilon(t)}=\int_{0}^{\xi} d t\left\{1+\frac{\varepsilon(t)}{\pi-\varepsilon(t)}\right\} \\
& \leq \xi+\frac{2}{\pi} \int_{0}^{\xi} \varepsilon(t) d t \leq \xi+3(\log 2+1)<\xi+6 .
\end{aligned}
$$

We deduce

$\mathrm{L}$ e m m a 8. If in the above mappings $s=\sigma+i \tau$ corresponds to $\zeta=\xi+i \eta$, where $\xi \geq-(1 / 2) \log 2$, then

$$
|\sigma-\xi|<A_{11} \text {. }
$$

We first take $\xi_{1}=-3 \pi-(1 / 2) \log 2, s_{2}=\xi_{2}=0$ in Lemma 1 . Then (2.4) yields

$$
-\sigma_{1}<A_{12}
$$

in view of (5.10) and (2.2). Also $s_{2}=\xi_{2}=0$, so that $\sigma_{1}<0$. Next we take $\xi_{2} \geq-(1 / 2) \log 2, \zeta_{2}=\xi_{2}+i \eta_{2}$. Then (2.3) and (2.4) yield

$$
\left|\left(\sigma_{2}-\sigma_{1}\right)-\pi \int_{\xi_{1}}^{\xi_{2}} \frac{d t}{\vartheta(t)}\right|<A_{13}
$$

Using (5.12) we deduce

$$
\left|\sigma_{2}-\sigma_{1}-\xi_{2}\right|<A_{14}
$$

i.e. $\left|\sigma_{2}-\xi_{2}\right|<A_{11}$, in view of (5.14). This proves Lemma 8 .

We now recall (5.1), (5.5) and define

$$
s=\log \frac{1+w}{1-w}, \quad w=\frac{e^{s}-1}{e^{s}+1}, \quad z=\varphi(w) .
$$

Clearly $|w|<1$, corresponds to $D_{2}$ in the $Z$ plane and so to $D_{0}$ in 
$|z|<1$. Also $\varphi(0)=1 / e$ and $\varphi(1)=1$. Let $f(z)$ be the function of Theorem 5. We consider

$$
\log ^{+}|f(z)|=u(z)=u\{\varphi(w)\}
$$

as a function of $w$ in $|w| \leq 1$. We write $u=U(w)$. Then

$$
\begin{aligned}
& \frac{1}{2 \pi} \int_{-\pi}^{\pi} U^{+}\left(e^{i \vartheta}\right) d \vartheta \\
& =\frac{1}{2 \pi} \int_{-\infty}^{+\infty}\left[U^{+}\left\{w\left(\sigma+\frac{i \pi}{2}\right)\right\}+U^{+}\left\{w\left(\sigma-\frac{i \pi}{2}\right)\right\}\right] \frac{d \sigma}{\cosh \sigma}
\end{aligned}
$$

We now return to the $\zeta$ plane. By (1.8) we have

$$
U^{+}(z) \leq k(r), \quad|z|=r .
$$

We write $K(r)=k(r) /\left(100 J^{2}\right)$ where $J$ is given by (1.9) and set

$$
k_{n}=K\left\{\exp \left(-2^{-n}\right)\right\} .
$$

We deduce that at all points $Z=X+i Y$, where $X \geq X_{n}$, we have $u \leq(10 J)^{2} k_{n}$. In particular if the points $\zeta=\xi_{n} \mp i \eta_{n}$ correspond to $s=\sigma_{n} \mp i \pi / 2$, we have for $n \geq 1$

$$
\begin{aligned}
& \int_{\sigma_{n-1}}^{\sigma_{n}} U^{+}\left\{w\left(\sigma \mp i \frac{\pi}{2}\right)\right\} \frac{d \sigma}{\cosh \sigma} \leq(10 J)^{2} k_{n} \int_{\sigma_{n-1}}^{\sigma_{n}} \frac{d \sigma}{\cosh \sigma} \\
& \leq 200 J^{2} k_{n} \int_{\sigma_{n-1}}^{\sigma_{n}} e^{-\sigma} d \sigma \leq 200 J^{2} k_{n} e^{-\sigma_{n-1}} \leq A J^{2} k_{n} e^{-\xi_{n-1}} \\
& =A J^{2} k_{n}\left(X_{n-1}^{2}+Y_{n-1}^{2}\right)^{1 / 2} \leq A J^{2} k_{n} Y_{n-1}
\end{aligned}
$$

in view of (5.13) and (5.2).

We set $r_{n}=\exp \left(-2^{-n}\right)$ and deduce that, for $n \geq 0,1-r_{n}=$ $\left(1-r_{n+1}\right)\left(1+r_{n+1}\right)>1.6\left(1-r_{n+1}\right)$. Thus

$$
\begin{aligned}
& \int_{r_{n}}^{r_{n+1}} \sqrt{\frac{K(r)}{1-r}} d r \geq \sqrt{k_{n}} \int_{r_{n}}^{r_{n+1}} \frac{d r}{\sqrt{1-r}} \\
& \quad=2 \sqrt{k_{n}}\left\{\left(1-r_{n}\right)^{1 / 2}-\left(1-r_{n+1}\right)^{1 / 2}\right\}>\frac{1}{5}\left(k_{n} 2^{-n}\right)^{1 / 2} .
\end{aligned}
$$

Further 


$$
\int_{0}^{1}\left(\frac{K(r)}{1-r}\right)^{1 / 2} d r=\frac{1}{10 J} \int_{0}^{1}\left(\frac{k(r)}{1-r}\right)^{1 / 2} d r=\frac{1}{10}
$$

Thus

$$
\sum_{0}^{\infty}\left(k_{n} 2^{-n}\right)^{1 / 2} \leq \frac{1}{2} .
$$

We now choose $Y_{n}=\inf \left\{1,\left(2^{n-1} k_{n+1}\right)^{-1 / 2}\right\}, n \geq 2$. Then $Y_{n}$ decreases with increasing $n$ and if $Y_{n}=1, n \leq n_{0}, Y_{n}<1, n>n_{0}$, we have

$$
\sum_{2}^{\infty} \frac{X_{n}}{Y_{n}} \leq \sum_{2}^{n_{0}} 2^{-n}+\sum_{n_{0}+1}^{\infty}\left(\frac{k_{n+1}}{2^{n+1}}\right)^{1 / 2} \leq \frac{1}{2}+\frac{1}{2}=1
$$

so that (5.2) holds. Also in view of (5.16) to (5.18) we have

$\frac{1}{2 \pi J^{2}} \int_{-\pi}^{\pi} U^{+}\left(e^{i \vartheta}\right) d \vartheta \leq A\left\{k_{0}+2 \sum_{n=1}^{\infty} k_{n} Y_{n-1}\right\} \leq A \sum_{0}^{\infty}\left(k_{n} / 2^{n}\right)^{1 / 2}<A_{15}$.

This proves (1.14) with $C_{2}=A_{15} J^{2}$ when $\vartheta=0$. The general case follows, since $f\left(z e^{i \vartheta}\right)$ satisfies the hypotheses of Theorem 2, whenever $f(z)$ does.

Next we recall that $\varphi(w)$, given by (5.15) maps $|w|<1$ into $|z|<1$ and $\varphi(1)=1, \varphi(0)=r_{0}=1 / e$ as required. Finally we have for $r_{0} \leq z<1$, using (5.13), (5.15)

$$
1-w>e^{-\sigma}>e^{-\xi-A_{11}}=A Z=A|\log z|>A(1-z) .
$$

This proves (1.13) and completes the proof of Theorem 5.

6. Proof of Theorems 6 and 2. To complete our results we need to establish the relation between the representation (1.2) and 1.b.c. We have

L e m m a 9. Suppose that $g(z)$ in (1.1) satisfies

$$
\int_{0}^{2 \pi}\left|u\left(r e^{i \vartheta}\right)\right| d \vartheta \leq C, \quad 0<r<1 .
$$

Then

$$
\int_{0}^{1}\left|v\left(\varrho e^{i \vartheta}\right)\right| \frac{d \varrho}{\varrho} \leq C, \quad 0 \leq \vartheta \leq 2 \pi .
$$


It is enough to assume $\vartheta=0$ in (6.2) since we may consider $g\left(z e^{i \vartheta}\right)$ instead of $g(z)$. We recall that (6.1) implies that $g(z)$ possesses the representation (1.2) in view of Theorem 1 and (1.6) shows that $\mu(\varphi)$ is a function of bounded variation, whose total variation in $[0,2 \pi]$ is at most $C$ by (6.1). We deduce from (1.2)

$$
v(\varrho)=\frac{-1}{2 \pi} \int_{-\pi}^{\pi} \frac{2 \varrho \sin \vartheta}{1-2 \varrho \cos \vartheta+\varrho^{2}} d \mu(\vartheta) .
$$

Thus

$$
\begin{aligned}
\int_{0}^{1}|v(\varrho)| \frac{d \varrho}{\varrho} & \leq \frac{1}{\pi} \int_{-\pi}^{\pi}|d \mu(\vartheta)| \int_{0}^{1} \frac{|\sin \vartheta|}{1-2 \varrho \cos \vartheta+\varrho^{2}} d \varrho \\
& =\frac{1}{\pi} \int_{-\pi}^{\pi}|d \mu(\vartheta)|\left|\left[\tan ^{-1} \frac{\varrho-\cos \vartheta}{\sin \vartheta}\right]_{0}^{1}\right| \\
& \leq \int_{-\pi}^{\pi}|d \mu(\vartheta)| \leq C .
\end{aligned}
$$

This proves Lemma 9 .

We have next

$\mathrm{L} \mathrm{e} \mathrm{m} \mathrm{m}$ a 10. If $g(z)$ satisfies the hypotheses of Theorem 6 then

$$
\int_{0}^{1}\left|v\left(\varrho e^{i \vartheta}\right)\right| \frac{d \varrho}{\varrho} \leq C_{2} C_{3}=16 C_{2} C_{1}\left[\pi+\frac{2}{\left(1-r_{0}\right)^{2}}\left(1+32 \pi C_{1}\right)\right] \text {. }
$$

It is again enough to consider the case $\vartheta=0$. We consider the function

$$
G(w)=g\{\varphi(w)\}=U+i V .
$$

It follows from (1.14) that

$$
\frac{1}{2 \pi} \int_{0}^{2 \pi} U^{+}\left(r e^{i \vartheta}\right) d \vartheta \leq C_{2}, \quad 0<r<1 .
$$

Also

$$
\frac{1}{2 \pi} \int_{0}^{2 \pi} U\left(r e^{i \vartheta}\right) d \vartheta=U(0)=u\left(r_{0}\right)
$$

Thus 
$\frac{1}{2 \pi} \int_{0}^{2 \pi}\left|U\left(r e^{i \vartheta}\right)\right| d \vartheta=\frac{1}{2 \pi} \int_{0}^{2 \pi}\left\{2 U^{+}\left(r e^{i \vartheta}\right)-U\left(r e^{i \vartheta}\right)\right\} d \vartheta \leq 2 C_{2}-u\left(r_{0}\right)$.

We now set $G_{1}(w)=G(w)-G(0)=U_{1}(w)+i V_{1}(w)$ and deduce that

$$
\frac{1}{2 \pi} \int_{0}^{2 \pi}\left|U_{1}\left(r e^{i \vartheta}\right)\right| d \vartheta \leq 2 C_{2}+2\left|u\left(r_{0}\right)\right| .
$$

Now Lemma 9 shows that

$$
\int_{0}^{1}\left|V_{1}(r)\right| \frac{d r}{r}=\int_{0}^{1}|V(r)-V(0)| \frac{d r}{r} \leq 4 \pi\left(C_{2}+\left|u\left(r_{0}\right)\right|\right) .
$$

Hence

$$
\int_{0}^{1}|V(r)-V(0)| d r \leq 4 \pi\left(C_{2}+\left|u\left(r_{0}\right)\right|\right)
$$

and so

$$
\int_{0}^{1}|V(r)| d r \leq 4 \pi\left(C_{2}+\left|u\left(r_{0}\right)\right|\right)+|V(0)| \leq 4 \pi\left(C_{2}+2\left|g\left(r_{0}\right)\right|\right) .
$$

We now note that

$$
V(r)=v(\varrho)
$$

where

$$
\varrho=\varphi(r) \text {. }
$$

It also follows from Pick's Lemma and (1.13) that

$$
\left|\frac{d \varrho}{d r}\right|=\left|\varphi^{\prime}(r)\right| \leq \frac{1-\varrho^{2}}{1-r^{2}} \leq 2 \frac{1-\varrho}{1-r} \leq 2 C_{1}, \quad 0<r<1 .
$$

Thus (6.4) yields

$\int_{r_{0}}^{1}|v(\varrho)| d \varrho=\int_{0}^{1} V(r)\left|\frac{d \varrho}{d r}\right| d r \leq 2 C_{1} \int_{0}^{1}|V(r)| d r \leq 8 \pi C_{1}\left(C_{2}+2\left|g\left(r_{0}\right)\right|\right)$.

Let $r_{1}=\max \left(r_{0}, 1 / 2\right)$. Then we deduce that 


$$
\int_{r_{1}}^{1}|v(\varrho)| \frac{d \varrho}{\varrho} \leq 16 \pi C_{1}\left(C_{2}+2\left|g\left(r_{0}\right)\right|\right) .
$$

We next deduce from (6.3) and Poisson's formula that

$$
U^{+}(t) \leq \frac{r+t}{r-t} \frac{1}{2 \pi} \int_{0}^{2 \pi} U^{+}\left(r e^{i \vartheta}\right) d \vartheta \leq C_{2} \frac{r+t}{r-t}, \quad 0<t<r .
$$

On letting $r$ tend to one we deduce

$$
U^{+}(t) \leq C_{2} \frac{1+t}{1-t} \leq \frac{2 C_{2}}{1-t}, \quad 0 \leq t<1 .
$$

Using (1.13) we deduce that

$$
u^{+}(\varrho) \leq \frac{2 C_{1} C_{2}}{1-\varrho}, \quad r_{0} \leq \varrho<1 .
$$

A corresponding inequality holds for $U^{+}\left(\varrho e^{i \vartheta}\right), 0 \leq \vartheta \leq 2 \pi$. We choose $\varrho=r_{2}=(1 / 2)\left(1+r_{1}\right)$ and apply Carathéodory's inequality to $g(z)$. This gives

$$
|g(r)| \leq \frac{2 r C_{1} C_{2}}{\left(1-r_{2}\right)\left(r_{2}-r\right)}, \quad 0 \leq r<r_{2} .
$$

Thus

$$
\begin{aligned}
\int_{0}^{r_{1}}|v(\varrho)| \frac{d \varrho}{\varrho} & \leq \int_{0}^{r_{1}}|g(\varrho)| \frac{d \varrho}{\varrho} \leq \frac{2 C_{1} C_{2}}{\left(1-r_{2}\right)\left(r_{2}-r_{1}\right)} \\
& =\frac{8 C_{1} C_{2}}{\left(1-r_{1}\right)^{2}} \leq \frac{32 C_{1} C_{2}}{\left(1-r_{0}\right)^{2}}
\end{aligned}
$$

Also

$$
\left|g\left(r_{0}\right)\right| \leq \frac{2 C_{1} C_{2}}{\left(1-r_{2}\right)\left(r_{2}-r_{0}\right)} \leq \frac{32 C_{1} C_{2}}{\left(1-r_{0}\right)^{2}} .
$$

On combining (6.5) to (6.7) we deduce Lemma 10 .

6.1. We can now complete our proofs. We recall the notation of the introduction. Then, with the hypotheses of Theorem 6 ,

$$
\left|U\left(r e^{i \vartheta}\right)\right|=\left|\int_{0}^{r} \frac{v\left(\varrho e^{i \vartheta}\right) d \varrho}{\varrho}\right| \leq C_{2} C_{3}
$$


in view of Lemma 10. Thus

$$
\begin{aligned}
I\left(r, \varphi_{1}, \varphi_{2}\right) & =\left|U\left(r e^{i \varphi_{2}}\right)-U\left(r e^{i \varphi_{1}}\right)\right| \\
& \leq 2 C_{2} C_{3}, \quad 0 \leq r<1,0 \leq \varphi_{1}<\varphi_{2} \leq 2 \pi .
\end{aligned}
$$

Also for fixed $\varphi_{1}, \varphi_{2}$

$$
I\left(r, \varphi_{1}, \varphi_{2}\right) \rightarrow \lim _{r \rightarrow 1}\left\{U\left(r e^{i \varphi_{2}}\right)-U\left(r e^{i \varphi_{1}}\right)\right\}=\int_{0}^{1} \frac{\left\{v\left(r e^{i \varphi_{2}}\right)-v\left(r e^{i \varphi_{1}}\right)\right\} d r}{r} .
$$

We set

$$
\mu(\varphi)=\int_{0}^{1} \frac{v\left(r e^{i \varphi}\right) d r}{r}
$$

and see that the hypotheses of Theorem 1 hold with $M=C_{2} C_{3}$. Further the limit (1.6) exists for every pair $\varphi_{1}, \varphi_{2}$. Thus we have proved Theorem 6. Now Theorem 2 follows at once from Theorems 5 and 6 . For in view of Theorem 5 we may apply Theorem 6 with $r_{0}=1 / e, C_{1}=A_{16}$ and $C_{2}=A_{15} J^{2}$. Thus, by Theorem 6, $g(z)$ has the representation (1.2) with $|\mu(\vartheta)| \leq A_{17} J^{2}$. We have already seen that (1.6) holds for every pair $\varphi_{1}, \varphi_{2}$.

\section{References}

[1] AHLFORs, L.: Untersuchungen zur Theorie der konformen Abbildung und der ganzen Funktionen. - Acta Soc. Sci. Fenn. Nova Series A 1:9, 1930, 1-40.

[2] Duren, P. L.: Theory of $H^{p}$ spaces. - Pure and Applied Mathematics 38. Academic Press, New York-London, 1970.

[3] Hayman, W. K.: On Nevanlinna's second theorem and extensions. - Rend. Circ. Mat. Palermo (2) 2, 1954, 346-392.

[4] Korenblum, B.: An extension of the Nevanlinna theory. - Acta Math. 135, $1975,187-219$.

[5] LEHTO, O., and K. I. VIRTANEN: Boundary behaviour of normal meromorphic functions. - Acta Math. 97, 1957, 47-65.

[6] Linden, C. N.: Functions regular in the unit circle. - Proc. Cambridge Philos. Soc. 52, 1956, 49-60.

Imperial College

Department of Mathematics

London S. W. 7

England
Tel-Aviv University

Department of Mathematics

Ramat-Aviv, Tel-Aviv

Israel

Received 22 August 1975 